\title{
Sobre la solución coherente de casos jurídicos
}

\author{
On Coherent Solutions to Legal Cases
}

\author{
Natalia Scavuzzo* \\ "The aim of philosophy is the clarification of the forms of \\ sense that, in one way or another, \\ are conceptually puzzling -for they are legion". \\ Hacker, P.M.S., Analytic Philosophy: \\ Beyond the Linguistic Turn and Back Again
}

Recepción: 06/08/2019

Evaluación: 14/11/2019

Recepción y aceptación final: 25/11/2019

\begin{abstract}
Resumen: Este trabajo consiste en un balance de la discusión presentada en Discusiones X acerca de "Diez tesis sobre la coherencia en el derecho" de Amalia Amaya. En primer lugar, se expone brevemente la teoría de la coherencia propuesta por Amaya y se indaga en forma crítica respecto del concepto de coherencia que la autora propone. Seguidamente se analiza cómo se relaciona el modelo de la coherencia con la discrecionalidad en la solución de casos jurídicos, cómo se conjuga un concepto de coherencia formal con el razonamiento no instrumental y qué perplejidades derivan del enfoque filosófico naturalista adoptado por Amaya. Por último, se argumenta que el modelo de la explicación y de la justificación coherentista debe explicitar el punto de vista relevante desde el cual se coherentiza.
\end{abstract}

Palabras claves: coherencia, razonamiento jurídico, identificación del derecho, justificación de decisiones judiciales

* Investigadora becada por la Università degli studi di Genova, Italia. Magíster en Derecho y Argumentación Jurídica, UNC, Argentina. Doctora en Filosofía del Derecho y Bioética Jurídica por la Università degli studi di Genova, Italia. Correo electrónico: nataliascavuzzo@hotmail.com. Agradezco a Alejandro Calzetta, Diego Dei Vecchi y al revisor anónimo por los valiosos comentarios a la primera versión de este trabajo. 
Natalia Scavuzzo

\begin{abstract}
This text the author offers an assessment of the debate carried out in Discusiones X about Amalia Amaya's "Diez tesis sobre la coherencia en el derecho". First, the author briefly explains Amaya's theory of coherence and critically analizes Amaya's concept of coherence. Next, the author analyzes how the model of coherence is related to discretion in the solution of legal cases, how a concept of formal coherence is combined with non-instrumental reasoning and what perplexities derive from the naturalistic philosophical approach adopted by Amaya. Finally, it is argued that the model of coherent explanation and justification must make explicit which is the relevant point of view from which the argument from coherence is developped.
\end{abstract}

Keywords: coherence, legal reasoning, identification of law, justification of judicial decisions.

\title{
1. Introducción
}

En este trabajo se busca retomar el debate en torno a las tesis que Amalia Amaya presenta en Diez tesis sobre la coherencia en el derecho ${ }^{1}$. El momento para retomar la discusión resulta más que oportuno, ya que la "coherencia" ha adquirido una atención creciente por parte de la cultura jurídica en los últimos años. En ese artículo Amaya introduce diez tesis fundamentales sobre la coherencia, que constituyen una síntesis de su propia teoría de la coherencia en el derecho ${ }^{2}$.

La autora propone una teoría general de la coherencia, cuya construcción se basa en el análisis de la coherencia en distintos ámbitos (ética, epistemología, filosofía del lenguaje, psicología) para construir un concepto unitario de coherencia aplicable al dominio del derecho. Entiende que su teoría es superadora de las teorías de la coherencia que precedentemente

1 El artículo fue publicado en la revista Discusiones X, en el año 2010. Participaron con comentaristas a este trabajo: Manuel Atienza, María Cristina Redondo, Guillermo Lariguet, Claudio Michelon, Aldo Schiavello y Juliano Maranhao.

2 Una presentación detallada de la teoría puede encontrarse en Amaya, A., The Tapestry of Reason. An Inquiry into the Nature of Coherence and its Role in Legal Argument, Oxford, Hart Publishing, 2015. 
se encontraban en el ámbito del derecho, atento a que su propuesta puede ser aplicada tanto al razonamiento sobre los hechos como al razonamiento sobre el derecho.

En este contexto, desarrolla un modelo de razonamiento jurídico, basado en la idea de coherencia, que consta de cuatro elementos:

1. Un concepto de coherencia jurídica en términos de satisfacción de restricciones.

2. Una explicación de las inferencias jurídicas basadas en la coherencia como una inferencia a la mejor explicación.

3. Una visión de la justificación jurídica como coherencia óptima.

4. Un enfoque contextualista a los estándares coherentistas de justificación ${ }^{3}$.

En el análisis de estas tesis sobre la coherencia en el derecho, emergen muchos de los temas tradicionales de la teoría del derecho ( $\mathrm{y}$ algunos que podríamos discutir si son o no parte de la teoría del derecho, o en qué medida lo son), a saber: racionalidad, interpretación, teorías de la verdad, distinción entre explicación y justificación, distinción entre reglas y principios, razonamiento jurídico, conflictos entre derechos, discrecionalidad judicial, justificación de las decisiones jurídicas, el uso de instrumentos lógicos-semióticos para la resolución de problemas jurídicos, la relación entre el derecho y la moral, entre el derecho y el razonamiento práctico, entre otros. Es por esto que la propuesta de Amaya constituye una compleja elaboración que nos abre distintos ámbitos de análisis y nos sitúa ante un panorama más que complejo.

En la discusión que este trabajo revisita se han señalado ambigüedades en el uso que la autora hace del término coherencia, probablemente derivadas de su intento de realizar una macro-teoría, y también algunas tensiones en relación a diversos de los temas mencionados. Aunque, como resultado del balance, no es claro que la teoría de Amaya pueda superar estos escollos, creo que la misma constituye una rica elaboración, útil para retomar algunos problemas persistentes en la teoría del derecho. En esta 
sede me propongo exponer algunos puntos que considero controvertibles de la propuesta de Amaya.

En la sección 2 presento los aspectos generales de la teoría de la coherencia en el derecho de Amaya. En la sección 3 analizo cuál es el concepto de coherencia de la teoría propuesta. En la sección 4 indago respecto de qué se predica la coherencia y cuál es la relación entre esta teoría de la coherencia y la discrecionalidad judicial. En la sección 5 cuestiono la teoría por cuanto asume un concepto formal de coherencia que le permite justificar razonamientos respecto de los fines últimos. En la sección 6 cuestiono particularmente el enfoque filosófico naturalista adoptado. Por último, en la sección 7 sugiero, a modo de complemento, que una explicación/justificación coherentista debería explicitar el punto de vista desde el cual se construye la coherencia.

\section{Presentación de la teoría coherentista del razonamiento jurídico}

Amaya nos presenta una forma de coherentismo como una teoría de la justificación en el derecho ${ }^{4}$. La autora sostiene que su teoría es superadora de otros estudios sobre la coherencia por dos razones. En primer lugar, porque su teoría, a diferencia de las otras, ofrece un concepto de coherencia. En segundo lugar, porque propone una noción unitaria de coherencia que considera "útil para definir el concepto de coherencia que es relevante para la justificación tanto de enunciados fácticos como normativos en el derecho" 5 .

Para ofrecer una definición de coherencia ella se basa en la teoría de Paul Thagard. La coherencia es la propiedad de los elementos de un conjunto que satisfacen la mayor cantidad de restricciones positivas y negativas. A partir de un conjunto de elementos podemos distinguir en subconjuntos que satisfacen o que no satisfacen estas restricciones. Las restricciones indican relaciones de coherencia (explicación, deducción, facilitación, asociación) e incoherencia (inconsistencia, incompatibilidad, asociación negativa) entre 
los elementos del conjunto. Las restricciones que los elementos deben satisfacer varían según el dominio en cuestión, por lo cual esta noción de coherencia puede aplicarse a diversos dominios. Como cada dominio determina cuáles son las restricciones que deben satisfacerse, puede decirse, en primer lugar, que el contexto pasa a ocupar un lugar importante en la justificación coherentista. Y, en segundo lugar, que esto hace que la definición de coherencia se nos presente como formal, ya que prescinde de parte de su contenido, el cual se determina en cada dominio específico.

Puede verse cómo, entonces, con el término coherencia se indica las relaciones de coherencia y de incoherencia. Podemos decir que la coherencia es la propiedad de los elementos del dominio consistente en tener cierto tipo de relación con los otros elementos del dominio (i.e., una propiedad relacional).

Simultáneamente, Amaya presenta la coherencia como un tipo de razonamiento, o el resultado de un tipo de razonamiento. Explica que el razonamiento coherentista es un tipo de razonamiento explicativo y justificativo. Determinar la coherencia es ver cómo distinguir en un conjunto de elementos entre un subconjunto de elementos aceptados y un subconjunto de elementos rechazados. En el caso del derecho, el órgano decisor se plantea un posible número de soluciones a un caso jurídico y selecciona entre ellas la más coherente.

Señala que el razonamiento coherentista es un tipo de inferencia, es una inferencia a la mejor explicación. El razonamiento que se basa en la coherencia es un tipo de razonamiento explicativo, donde quienes toman las decisiones alcanzan creencias óptimamente coherentes sobre los hechos y el derecho mediante una inferencia a la mejor explicación.

Además, Amaya sostiene la tesis de que "el coherentismo (...) sí que cuenta con una clara descripción de los procesos de inferencia que resultan en creencias justificadas" 6 . Por esto, como dijimos, se trata de un razonamiento explicativo y justificativo. Se presenta como un tipo de razonamiento que puede justificar las decisiones en el derecho. Para lograr esto, explica que a la teoría de la coherencia debe unirse una teoría de la responsabilidad epistémica. La responsabilidad epistémica juega un rol relevante, en virtud de que Amaya reconoce que existe el riesgo de que la hipótesis 
más coherente sea aquella fruto de la manipulación de los elementos que ha hecho el decisor o producto de su formación de creencias irracionales.

El objetivo que Amaya se propone es construir una teoría de la coherencia psicológicamente plausible, pero también normativamente adecuada. Por esto, además, se complementa la teoría de la coherencia con una teoría de la responsabilidad epistémica. De esta forma, la responsabilidad epistémica está implícitamente contenida en la coherencia ${ }^{7}$.

La autora explica que la teoría de la coherencia como satisfacción de restricciones no aborda el problema esencial de cómo se genera el conjunto inicial de elementos que se consideran relevantes para comparar y evaluar la coherencia (problema del input). Ella acepta un tipo de coherencia "discriminating" 8 que favorece un conjunto de elementos para la aceptación, aun si al final la aceptación de estos elementos depende de su coherencia con todos los elementos, y que esta se diferencia de una teoría de la coherencia "pura" como la de Dworkin.

Es importante resaltar que este tipo de razonamiento coherentista se presenta como un razonamiento de tipo no-instrumental. Explica Amaya que es un razonamiento acerca de los fines, que es útil para resolver conflictos entre valores y fines que están presentes en el derecho y para realizar inferencias prácticas.

Creo finalmente que en la presentación de Amaya puede entenderse que la coherencia, además de ser una propiedad y una forma de razonamiento, es también un valor ${ }^{9}$. Encontramos que la autora ofrece diversas razones para adoptar un estándar coherentista de la justificación, estas son:

7 Amaya explica que la responsabilidad epistémica puede basarse en dos concepciones, una deóntica y otra de las virtudes. En "Diez tesis sobre la coherencia en el derecho" ella defiende como mejor alternativa para el contexto jurídico una posición intermedia que tiende a la concepción de las virtudes. Ibídem, p. 36-37. Sin embargo, luego en The Tapestry of Reason. An inquiry into the Nature of Coherence and its Role in Legal Argument, se inclina por aplicar al contexto jurídico una concepción de la responsabilidad epistémica basada en las virtudes, pero en la cual los deberes juegan un rol central. Véase Amaya, A., The Tapestry of Reason, op. cit., pp. 522-523.

8 Véase Amaya, A., The Tapestry of Reason, op. cit., p. 546.

9 Para una defensa de la coherencia como valor supremo en el derecho véase Pérez Bermejo J.M., "Alcune osservazioni sul valore della coerenza nei sistemi giuridici" en Diritto \& questioni pubbliche, 2007, pp. 56-58. 
(a) Epistémicas, ya que la coherencia y la verdad están conectadas de manera apropiada por la inferencia a la mejor explicación.

(b) Prácticas, ya que facilita la coordinación y la efectividad de acciones conjuntas, y la eficacia de ciertos valores o fines.

(c) Tiene valor constitutivo de la identidad individual y colectiva.

(d) Otra que podríamos llamar ideológica, ya que para Amaya el coherentismo es una vía intermedia entre el formalismo y el escepticismo.

(e) La última razón que presenta a favor del coherentismo es que "este goza de un alto grado de plausibilidad desde el punto de vista psicológico"10. Llamaré a esta, una razón naturalista.

\section{Sobre el concepto de coherencia, ¿una propiedad o un tipo de razonamiento?}

Como se explicó precedentemente, Amaya propone un concepto unitario de coherencia útil para ser aplicado tanto al razonamiento sobre los hechos como al razonamiento sobre el derecho, sin embargo la coherencia es a la vez una propiedad (relacional), un tipo de razonamiento (o el resultado de un tipo de razonamiento) y un valor. Esta ambigüedad también está presente en las razones ofrecidas en favor del modelo coherentista. Algunas de ellas son razones a favor de adoptar un razonamiento de tipo choerentista, otras justifican adoptar una decisión óptimamente coherente, y otras son razones que justifican porqué es valiosa la coherencia.

La razón epistémica, según la cual la coherencia y la verdad están conectadas mediante la inferencia a la mejor explicación, considera a la coherencia como un tipo de razonamiento explicativo ${ }^{11}$. Por otro lado, la razón práctica, según la cual la coherencia facilita la coordinación y la eficacia de ciertos valores, considera a la coherencia como una propiedad. Mientras que la razón ideológica, según la cual la coherencia permite supe-

${ }^{10}$ Amaya, A., "Diez tesis sobre la coherencia en el derecho", op. cit., p. 58.

${ }^{11}$ De una forma u otra, todos los comentarios presentes en el número de discusiones han puesto en discusión la relación entre la coherencia y la verdad. Amaya reconoce que esta relación es más que problemática, pero no da argumentos para sostenerla. 
rar al formalismo y al escepticismo, parece suponer ambos conceptos de coherencia a la vez, a la coherencia como una propiedad y a la coherencia como un tipo de razonamiento.

Amaya defiende que su teoría de la coherencia es una vía intermedia entre el formalismo y escepticismo. No obstante, no es claro en este contexto cómo se entienden el formalismo y el escepticismo ${ }^{12}$. De esta forma, la ambigüedad señalada en concepto de coherencia se traslada a este debate.

El formalismo puede entenderse como una tesis según la cual la aplicación de reglas es una cuestión de inferencias lógicas que se expresan en verdades analíticas. En este contexto el formalismo se ve como una posición que defiende la posibilidad de una justificación formal de las decisiones judiciales (justificada por la lógica formal) en virtud de ciertas premisas que se tienen por válidas y ciertas reglas de inferencia ${ }^{13}$. En esta concepción el formalismo es una teoría sobre el razonamiento.

En lo que respecta al escepticismo, si bien puede interpretarse como una teoría anti-formalista que niega la posibilidad de que la lógica formal pueda aplicarse a directivas o proposiciones y, en este sentido, también concebirse como una teoría sobre el razonamiento jurídico, por lo general son llamados "escépticos" quienes niegan que las fuentes del derecho expresen normas que pueden conocerse (escépticos en materia de interpretación). Otra forma de anti-formalismo (que en algún sentido también sería una suerte de escepticismo) está dada por quienes niegan que los sistemas jurídicos posean la propiedad de ser coherentes o que pueda haber siempre una respuesta correcta que derive de estos sistemas (entendida como la respuesta coherente).

12 Explica Tarello cómo la locución "Formalismo Jurídico" se ha presentado en la cultura jurídica de forma ambigua. Entre los diversos sentidos que se han atribuido pueden nombrarse: formalismo como característica de ciertos ordenamientos jurídicos; como característica o vicio profesional de los juristas; como concepción del derecho que lo entiende como una "forma" de la experiencia que distingue lo jurídico de lo no jurídico; a la ciencia del derecho como una ciencia formal; como una concepción de la interpretación. En este sentido las corrientes que se oponen al formalismo también son de diverso tipo, y como bien señala Tarello, es posible ser formalista en un sentido y no en otro. Véase, Tarello, G., "Formalismo", Novissimo digesto italiano, Torino, UTET, vol. VII, pp. 571-580.

${ }^{13} \mathrm{Al}$ respecto véase, Wróblewski, J., "Justificación de las decisiones jurídicas" en Sentido y hecho en el derecho, México, Fontamara, 2001, pp. 45- 65. 
Sobre la solución coherente de casos jurídicos

Es por esto que, por una parte, el coherentismo de Amaya en cuanto entiende la coherencia como una forma de razonamiento se opone a las teorías que tratan de aplicar la lógica formal al razonamiento jurídico. De esta forma podemos decir que estamos ante dos modelos diversos de razonamiento en el derecho.

Pero, por otra parte, esta propuesta coherentista parece rechazar al escepticismo no ya como teoría sobre el razonamiento jurídico, sino como teoría sobre las propiedades de los sistemas jurídicos. En este sentido, Amaya remarca el fracaso del positivismo formal de construir una teoría pura del derecho,

El fracaso del proyecto del positivismo formal de ofrecer una teoría pura del derecho no nos aboca a aceptar las conclusiones escépticas que caracterizan a los realistas jurídicos y (en distinto grado, sin duda) a los movimientos críticos, sino a repensar el modelo de racionalidad que es apropiado para el derecho, en un esfuerzo por desarrollar una concepción amplia de la razón en el Derecho, más allá de los límites impuestos por los modelos formalistas ${ }^{14}$.

Nuevamente, diversas tesis pueden entenderse como una forma de formalismo, pero es llamativa la identificación de "positivismo formal" con su referencia a una teoría pura del derecho ${ }^{15}$. Esto resulta extraño y creo que sería oportuno revistar cuál es el objeto de la teoría de Kelsen (al menos en La teoría pura del derecho) que es asimilada a alguna forma de formalismo ${ }^{16}$.

Para Kelsen el objeto de La teoría pura del derecho es el conocimiento del derecho. Respecto al método sostiene que es necesario eliminar todo lo que no corresponda exactamente a su objeto de estudio. De esta forma, Kelsen

${ }^{14}$ Véase Amaya A., "Diez tesis sobre la coherencia en el derecho", op. cit., p. 56.

${ }^{15} \mathrm{Al}$ respecto, por ejemplo, Marmor explica que ningún positivista diría que la aplicación de reglas es una cuestión de inferencia lógica. Véase Marmor, A., Interpretation and Legal Theory, 2da ed., Hart Publishing, 2005, p. 98.

${ }^{16} \mathrm{Al}$ respecto explica Tarello que la teoría de Kelsen puede entenderse formal en diversos sentidos: porque funda la posibilidad del discurso jurídico en una "forma" categorial; porque prescinde del contenido y se refiere a la naturaleza de cualquier posible ordenamiento 
busca delimitar el conocimiento del derecho del conocimiento de otras cuestiones, es decir, quiere precisar cuál es el objeto de estudio. Kelsen no quiere negar que otras cuestiones como, por ejemplo, las cuestiones políticas sean importantes, o que estén íntimamente relacionadas con el derecho, sino que busca evitar el sincretismo metodológico que para él oscurece la esencia de la ciencia jurídica ${ }^{17}$. Kelsen no presenta una teoría formalista del razonamiento jurídico, porque la teoría pura no es una teoría del razonamiento jurídico o una teoría sobre la justificación de decisiones jurídicas ${ }^{18}$.

Por otro lado, en relación a "las conclusiones escépticas", corresponde precisar que el escepticismo, si se entiende como una forma de escepticismo interpretativo (lo que caracteriza a algunos de los múltiples realismos jurídicos), no sostiene una tesis escéptica en relación al razonamiento jurídico, sino respecto de la posibilidad de determinación del ordenamiento jurídico ${ }^{19}$. Esta forma de escepticismo no negaría que existen instrumentos interpretativos que tienden a la coherencia ${ }^{20}$ y que los intérpretes usan la coherencia como razón para elegir entre distintas interpretaciones. Lo que sí negaría es que sea posible predicar la coherencia de los sistemas jurídicos.

Amaya construye una teoría del razonamiento coherentista donde las decisiones están justificadas porque satisfacen óptimamente las restricciones negativas y positivas del contexto específico. Sin embargo, señala que el

jurídico; o porque establece criterios formales para la determinación de que una norma existe. Tarello, G., op. cit., p. 572.

17 Véase Kelsen H., Teoría Pura del derecho, Buenos Aires, Ediciones Colihue, 2011.

${ }^{18}$ En este sentido señala Wróblewski: "Hoy nadie sigue creyendo en una aplicación del derecho puramente mecánica al estilo de los positivistas del siglo XIX, de la Begriffsjurisprudenz. Ese rechazo de una idea falsa no implica, sin embargo, que se denuncie también la ideología de la legalidad; pero, el que la mantenga estará obligado a justificarla de otra forma." Wróblewski, J., "La interpretación en el derecho: teoría e ideología" en Sentido y hecho en el derecho, México, Fontamara, 2001 [1979], pp. 149- 171, p. 150.

$19 \mathrm{Al}$ respecto véase Guastini, R., Interpretar y Argumentar, Madrid, Centro de Estudios Políticos y Constitucionales, 2014.

${ }^{20}$ Por ejemplo, Guastini señala que la "interpretación sistemática” incluye una familia de argumentos interpretativos que tienen en común: referencia al contexto en que se encuentra la disposición a interpretar y al presunto carácter sistemático del ordenamiento jurídico. Explica que esta forma de argumentar esconde distintas operaciones interpretativas: combinar fragmentos de normas, interpretación según el sector del ordenamiento donde se encuentra, permanencia terminológica, o del cambio terminológico, interpretación adecuadora. Véase Guastini, R., Interpretar y Argumentar, op. cit., pp. 289-299. 
razonamiento coherentista no puede garantizar que la decisión en un caso particular esté justificada. Esto es así, porque el razonamiento coherentista consiste en una inferencia a la mejor explicación y la mejor explicación podría ser el fruto de una formación de creencias irracionales. La solución que propone a este problema es que la decisión más coherente sería aquella que un decisor racional hubiera alcanzado. Es por esto que Amaya sostiene que los estándares de responsabilidad epistémica, que caracterizan qué es un decisor racional, son un componente esencial de una teoría coherentista de la justificación.

En relación a este punto, se argumenta que las personas tendemos a buscar coherencia, por lo que es necesario considerar cómo diferenciar entonces la coherencia que produce justificación y la coherencia que es el resultado de prejuicios o creencias mal formadas. La autora responde a este punto que "una hipótesis acerca de los hechos o del derecho está justificada si podría ser el resultado de un proceso epistémicamente responsable de maximización de la coherencia"21. La decisión justificada (la respuesta correcta), no necesariamente es el resultado de una inferencia a la mejor explicación. La hipótesis óptimamente coherente es la solución que un decisor jurídico epistémicamente responsable podría haber aceptado en virtud de la coherencia. De allí que “...la justificación de una decisión jurídica depende de una condición contrafáctica, no causal...”22. La solución siguiendo el razonamiento coherentista es siempre derrotable.

Nuevamente, aquí puede verse como no distinguir entre coherencia como propiedad y coherencia como razonamiento oscurece el argumento y cómo la confusión entre ambas cosas puede traernos algún problema ${ }^{23}$.

La coherencia puede entenderse como un tipo de razonamiento o como el producto o resultado de este tipo de razonamiento. Sin embargo, en la propuesta de Amaya, la decisión más coherente no es necesariamente el producto o resultado de un razonamiento coherentista. La coherencia se predica de la solución a la que un agente, que satisface los requisitos de

${ }^{21}$ Véase Amaya, A., "Diez tesis sobre la coherencia en el derecho", op. cit., p. 35.

${ }^{22}$ Ibídem, p. 35. En el mismo sentido, Véase Amaya, A., The Tapestry of Reason, op. cit., p. 520.

${ }^{23}$ Redondo ha señalado este tensión, véase Redondo, M.C., "Decidir en modo justificado: una explicación coherentista", Discusiones, X, 2010. 
responsabilidad epistémica, hubiera podido acceder. Pero, Amaya reconoce, podría darse el caso de un agente epistémicamente responsable que sin seguir un procedimiento responsable de maximización de la coherencia llegue a la decisión más coherente ${ }^{24}$.

En particular, conforme esta propuesta no podemos saber si una decisión es coherente en virtud de que posee tal propiedad (cumple con la satisfacción de restricciones negativas y positivas óptimamente) o bien porque es adoptada mediante un razonamiento de tipo coherentista (es una inferencia a la mejor explicación). Por esto me pregunto, si la teoría de la coherencia analizada es una teoría explicativa y justificativa sobre el razonamiento jurídico o bien una teoría sustancial sobre cuál es la decisión justificada, independientemente de cómo esta sea alcanzada, i.e. la solución correcta a un caso particular que un decisor racional alcanzaría.

\section{Un viejo problema recurrente: discrecionalidad de los órganos decisores}

En la actualidad si bien es raro que se sostenga que los sistemas jurídicos son coherentes, sí es actual la idea de que los principios constitucionales imponen la coherentización de los sistemas jurídicos ${ }^{25}$. Estas propuestas sobre el rol de la coherencia, se apoyan en diversas versiones de coherentismo, según el cual los ordenamientos jurídicos tendrían naturaleza sistemática a partir de sus principios últimos expresos o no en los textos

24 Véase Amaya, A., The Tapestry of Reason, op. cit., p. 524, donde expresa: "the counterfactual version allows for the possibility that a legal decision be justified, provided that an epistemically responsible legal decision-maker could have accepted it as justified, even if it results from an irresponsible process of coherence maximization. This is important if we want to preserve, as we most certainly should, the crucial distinction between 'normative' reasons and 'explanatory' reasons, that is to say, the distinction between the reasons that support a decision and the reasons that explain why a particular decision was taken. That a right legal decision was taken for the wrong reasons does not in the least reduce its correctness". Podría considerarse que, bajo estas consideraciones, la responsabilidad epistémica pierde, al menos en parte, parte importante de la relevancia atribuida por la teoría.

${ }^{25}$ Ratti, G.B., "La coerentizzazione dei sistemi giuridici”, Diritto \& questioni pubbliche, 7, 2007, pp. 61-70. 
Sobre la solución coherente de casos jurídicos

constitucionales $^{26}$. En estas posiciones juega un rol central la distinción entre reglas y principios, siendo estos últimos los que permiten entender al derecho como un sistema ordenado (no así las reglas, que no tendrían un rol importante en el ordenamiento jurídico).

Sin embargo, la ambición de coherentizar el ordenamiento jurídico a través de sus principios últimos es más que problemática. Por un lado, como reconoce Amaya, en los ordenamientos jurídicos confligen diversos fines y valores, podemos agregar diversas ideologías, lo que se traduce en que es posible constatar en éstos inconsistencias axiológicas. Por otro lado, la distinción misma entre reglas y principios es más que dudosa ${ }^{27}$ (y en todo caso, dependiente de la interpretación ${ }^{28}$ ).

Como ya se dijo, Amaya en Diez tesis sobre la coherencia en el derecho reconoce que los sistemas jurídicos pueden ser incoherentes (que generalmente hay conflictos entre los valores y fines que protege el derecho), lo que debe ser coherente en su propuesta son los enunciados producto de la

${ }^{26}$ En este sentido es común hablar de consistencia axiológica. La coherencia se entiende como cohesión axiológica (coherence), es decir que las normas de un ordenamiento jurídico pueden ser reconducibles a un principio o conjunto de principios o valores (y de esto se seguiría que cada cuestión admite solo una respuesta correcta). Este sentido se distingue de la coherencia lógica entre las normas (consistency), es decir ausencia de antinomias. Véase Guastini, R., Interpretar y argumentar, op. cit., p. 286-287. Al respecto de la armonía entre los principios Guastini sostiene que "La armonía axiológica entre principios, en la medida en que existe, es fruto de construcción jurídica, es decir de la (ocasional) armonización de los mismos por obra de los juristas y los jueces" (Ibídem, p. 287).

27 Explica Hart en su Postscriptum a El concepto de derecho, que la distinción (al menos como presentada por Dworkin, entre reglas que son de aplicación todo o nada y principios no concluyentes) parece ser solamente una cuestión de grados. Véase, Hart, H.L.A., "Postscriptum” en Bonilla, D., et al. La Decisión Judicial. El debate Hart-Dworkin, Bogotá, Universidad de los Andes, Siglo del Hombre Editores, 1997, p. 122.

${ }^{28}$ Esta sería una consecuencia del escepticismo interpretativo, en este sentido, por ejemplo Riccardo Guastini sostiene que "hay que aclarar, por si acaso no fuese obvio, que el carácter fundamental o marginal de una norma, su carácter derrotable o inderrotable, y (en muchos casos) el carácter abierto o cerrado de un supuesto de hecho no son, digámoslo así, datos objetivos, anteriores a la interpretación: al contrario, dependen de la interpretación, son fruto de la interpretación (...) En consecuencia, la identificación de una norma como regla o como principio (la adscripción de una norma a la clase de las reglas o a la clase de los principios) depende de la interpretación”, en Interpretar y argumentar, op. cit., pp. 190-191. 
identificación de los hechos relevantes y del derecho aplicable y, a su vez, las decisiones de los jueces.

Pero, ¿cómo funciona exactamente la coherencia en el razonamiento jurídico? Frente a un sistema jurídico (generalmente incoherente), el juez elabora una hipótesis de decisión jurídica coherente mediante el razonamiento a la mejor explicación. Esto sirve tanto para los hechos, el derecho y la decisión en sí misma consideradas. Pensemos, por ejemplo, en los enunciados sobre el derecho aplicable. Mediante los mecanismos sugeridos por Amaya (contracción, adición y reinterpretación) se presenta como más coherente un enunciado respecto del derecho aplicable (i.e. identificación de los estándares normativos) mediante una inferencia que parece basarse en consideraciones sustantivas tomadas por el órgano decisor.

Generalmente, en los ordenamientos jurídicos modernos se exige que las decisiones sean motivadas (en el sentido de que deben darse razones que las justifiquen) en base al derecho ${ }^{29}$. Cuando la decisión no se basa en el derecho (caso de lagunas o antinomias) tradicionalmente se entiende que las decisiones son el producto de la discrecionalidad del órgano decisor.

La teoría de la coherencia propuesta justifica decisiones conforme a derecho, pero también decisiones en los casos en que el derecho no establece una solución o establece una solución contradictoria ${ }^{30}$. En este caso la justificación coherentista permitiría justificar decisiones que exceden la justificación conforme a derecho, en este sentido parece ocultarse que algunas decisiones, producto de una elección discrecional conforme a consideraciones sustantivas extra-jurídicas, no se encuentran efectivamente justificadas conforme a derecho. Aquí el peligro: en estos casos el recurso a la coherencia parece esconder una forma de retórica que disfraza de justificación jurídica a la elección discrecional de una decisión.

La sospecha de discrecionalidad latente en las teorías coherentistas también ha sido presentada por otros autores, por ejemplo, Schiavello argumenta que la construcción de la coherencia es altamente discrecional,

${ }^{29}$ Guastini, R., Interpretar y argumentar, op. cit. p. 231.

${ }^{30}$ Podría ser también cuando se dan los conflictos de instanciación (o antinomias en concreto). Que se caracterizan por una imposibilidad fáctica, no normativa, con es el caso de conflictos normativos (antinomias en abstracto). Al respecto véase Navarro, P.y Rodríguez J.L., Deontic Logic and Legal Systems, Cambridge Univertisy Press, Cambridge, 2014, p. 183. 
Sobre la solución coherente de casos jurídicos

y que la noción de responsabilidad epistémica es tan vaga que, cualquiera sea la concepción por la que se opte (deóntica o de la virtud), solo juega un papel retórico para apoyar la decisión preferida por el decisor en cada caso $^{31}$. Expresamente afirma:

Che le teorie coerentiste spaccino sotto le (mentite) spoglie di una attraente teoria del sistema giuridico una politica del diritto discutibile è un sospetto che merita di essere preso sul serio. Come sostiene in particolare il realismo giuridico, potrebbe darsi che le teorie coerentiste amplino surrettiziamente la discrezionalità dell'interprete e del giudice in particolare affermando di voler fare esattamente il contrario. Questo è laspetto più marcatamente idologico e retorico delle teorie coerentiste che è compito della scienza giuridica tentare di svelare ${ }^{32}$.

En el mismo sentido Ratti sostiene:

Sostenere che vi sia sempre una risposta correta basata su ragioni morali ultime, quando è più che dubbio che tali ragioni esistano, equivale a difendere l'oggettività di un punto di vista che è, nel migliore dei casi, politicamente accettabile, ma che certamente non è privo di punti discutibili. Sembra che il proposito del coerentismo, in effetti, sia quello di marginalizzare molte delle fonti giuridiche rilevanti, per lasciare che i giudici sistematizzino il diritto in conformità a regole morali (assertitamente) oggettive, la cui origine, tuttavia, rimane misteriosa ${ }^{33}$.

Amaya considera que estas críticas son infundadas ${ }^{34}$. Explica que su concepción de la coherencia no sostiene que existe una decisión correcta y, además, en respuesta a Schiavello sostiene que los “estándares [de res-

31 Véase Schiavello, A., "Tre osservazioni su Diez Tesis acerca de la coherencia en el derecho", Discusiones, $\mathrm{X}, 2010$.

32 Ibídem, pp. 161-162.

${ }^{33}$ Ratti G.B., op. cit., p.70

${ }^{34}$ Debe aclararse que la crítica de Schiavello se dirige directamente a la propuesta de Amaya, mientras que la de Ratti es una crítica general a las teorías de la coherencia. 
ponsabilidad epistémica] limitan el modo en el que se puede construir la coherencia en el curso de los procesos de decisión jurídica y no cumplen, por lo tanto, una función meramente retórica" ${ }^{35}$. Para Amaya estos criterios de responsabilidad epistémica no pueden legitimar cualquier decisión. Lo que esta noción de responsabilidad epistémica permite es excluir ciertas hipótesis como injustificadas.

El proceso orientado a la maximizaicón de la coherencia, no es un proceso que depende de la intuición y las elecciones valorativas del decisor. En este sentido sostiene Amaya:

según la concepción coherentista de la justificación jurídica defendida en "Diez Tesis", no es que haya una hipótesis coherente tal que el agente epistémicamente responsable tiene que individuarla, sino que una hipótesis está justificada en virtud de su coherencia si es una hipótesis que un agente epistémicamente responsable podría haber aceptado como tal $^{36}$.

Dada esta importancia que en la teoría juegan los criterios de responsabilidad epistémica, sería entonces más que necesario un análisis detallado de los mismos, que la autora reconoce como vagos ${ }^{37}$. Y, por sobre todo, la inquietud que surge es ¿cuáles son y cómo se seleccionan los elementos respecto de los que se establecerá la relación de coherencia? Amaya reconoce que un problema que se mantiene sin solución es el modo en que se crea el conjunto de los elementos iniciales que se creen relevantes para comparar la coherencia de las decisiones alternativas. En el contexto del derecho esto nos reenvía directamente al problema tradicional de la teoría del derecho de la identificación de los estándares normativos jurídicos y su independencia o no de otros estándares de conducta (a saber, por ejemplo, normas morales).

Nuevamente, en la teoría de Amaya la decisión que el órgano decisor debe tomar es aquella más coherente entre las otras posibles hipótesis de

35 Amaya, A., "Diez tesis sobre la coherencia en el derecho", op. cit., p. 247.

${ }^{36}$ Ibídem, p. 248. 
decisión. Específicamente Thagard explica que en un problema jurídico los elementos del dominio son los principios del derecho y las decisiones de la corte ${ }^{38}$. Afirma que: "To show that a given problem is a coherence problem in this sense, it is necessary to specify the elements and constraints, provide an interpretation of acceptance and rejection, and show that solutions to the given problem do in fact involve satisfaction of the specified constraints"39.

Por lo tanto, la coherencia es una propiedad de una decisión que un sujeto epistémicamente responsable podría tomar, que satisface más que otras decisiones los requerimientos de los principios del derecho. $\mathrm{Al}$ respecto, y a los fines de preservar la idea de juzgar conforme a derecho, es interesante la propuesta coherentista que presenta Juan Pablo Alonso respecto de la posibilidad de usar la coherencia dentro de una teoría positivista del derecho. Alonso sostiene que solamente los principios reconocidos por un ordenamiento jurídico específico pueden servir de guía para resolver de la forma más coherente algunos casos de lagunas normativas ${ }^{40}$.

Amaya parece reconocer que el derecho tiene una posibilidad limitada de justificar decisiones. Sin embargo, también parecen presentarse como decisiones justificadas conforme a derecho, decisiones en base a sistemas de normas inconsistentes al introducir (de forma velada) la discrecionalidad dentro de la inferencia que justifica la decisión. Aun cuando recurrimos a los principios del derecho, que pueden presentarse en contradicción entre ellos y, por lo tanto, en estos casos la solución más coherente deriva también de una elección del decisor ${ }^{41}$.

38 Thagard, P. y Verbeurgt, K., "Coherence as Constraint Satisfaction", Cognitive Science, 22 (1), 1998, pp. 1-24.

${ }^{39}$ Ibíd. p. 4

40 Alonso, J. P., Principios Jurídicos Implícitos, Madrid, Marcial Pons, 2018 y Alonso, J.P., "Principios jurídicos implícitos y coherencia", Doxa, 36, 2013, pp. 375-385.

${ }^{41}$ Otras teorías de inspiración coherentistas, no es el caso de Amaya, recurren a la idea de la ponderación o balance para determinar cuál es el principio que en un caso concreto debe prevalecer para resolver un caso particular. Véase, por ejemplo, Alexy R., Teoría de los derechos fundamentales, Madrid, Centro de Estudios Constitucionales, 1993 [1986]. En la vereda de en frente, el realismo genovés explica que los decisores en tales circunstancias crean una jerarquía axiológica móvil para resolver los conflictos entre derechos. Véase Guastini, R., Interpretar y argumentar, op. cit. pp. 216-218. 
Que los órganos decisores otorgan a las fuentes del derecho significados que permiten prevenir antinomias o inconsistencias entre las normas y los principios fundamentales del ordenamiento o atribuyen a las fuentes significados que quepan mejor a sus intuiciones morales, no es una característica sorprendente para la teoría del derecho. Como ya mencioné, algunos realistas jurídicos enfatizan esta realidad respecto a la interpretación y aplicación de las fuentes del derecho. La diferencia con la teoría propuesta es clara, el realismo jurídico no ofrece una teoría normativa sobre la justificación de las decisiones judiciales.

Sin embargo, creo que un riesgo del modelo coherentista del razonamiento jurídico propuesto es que oscurece en parte la diferencia entre la justificación de una decisión en virtud de ciertas premisas que se tienen por válidas según ciertas reglas de inferencia aceptadas y la justificación, a su vez, de estas premisas (su bondad). Así una forma de preservar esta distinción en distinguir entre la justificación interna (relación de deducibilidad entre premisas según ciertas reglas aceptadas) y la justificación externa de una decisión judicial (justificación de las premisas de hecho y derecho) ${ }^{42}$.

El apoyo al razonamiento de tipo coherentista parece apoyarse en un rechazo (al menos parcial) de los instrumentos propios de la lógica formal. Sin embargo, cuando se aplican los principios de la lógica formal a un argumento, estos no garantizan de por sí la verdad de la conclusión. Lo único que la aplicación de las reglas de inferencias aceptadas garantizan es que si las premisas son verdaderas, entonces la conclusión lo será. En relación al derecho la lógica forma suele usarse en el denominado "silogismo judicial", en donde en base a las premisas de hecho y derecho se deduce una conclusión que constituye la decisión judicial. En la justificación de las premisas (de hecho y derecho) los argumentos aceptados son más amplios. En definitiva, es la justificación externa la encargada de justificar las premisas. Una decisión podría estar internamente justificada (en base a una relación de inferencia entre las premisas adoptadas), pero no estarlo externamente ${ }^{43}$ (porque dichas premisas no estaban adecuadamente

42 Wróblewski, J., The judicial aplication of the law, ed. por Bankowski, Z. y MacCormick, N., Kluwer, Dordrecht, 1992 [1972]. 
justificadas). Contrariamente, el modelo coherentista parece justificar una decisión sin distinguir entre la aceptabilidad de las premisas y la decisión tomada conforme a estas premisas.

Conforme estas consideraciones, podemos ver que el modelo de la coherencia, por un lado, sostiene no tratar el problema de la justificación externa o de aplicabilidad o bondad de las premisas de hecho y derecho, pero, por otro lado, justifica la decisión no solo en cuanto a su racionalidad interna sino también como la solución más coherente en base a decisiones sustanciales. En definitiva, como sostiene Wróblewski, en referencia a Perelman, no es descabellado sostener que la bondad de algunas premisas sea, después de todo, objeto de persuasión mediante L’empire rhétorique.

\section{Un concepto formal de coherencia y una teoría de la racionalidad sustancial}

El concepto de coherencia, como satisfacción de restricciones que dependen del dominio específico del que quiera predicarse que un elemento es coherente, se presenta como un concepto abstracto o, podríamos decir, formal ${ }^{44}$. En este sentido, cuáles sean las restricciones que deben satisfacerse depende contextualmente de cada dominio.

Según la teoría Amaya, las restricciones que deben satisfacerse en el derecho son las de coherencia deductiva, explicativa, analógica y deliberativa. En la premisa normativa agrega la coherencia interpretativa; y en la premisa fáctica a las coherencias conceptual y perceptual. Por otro lado, los elementos entre los que debe maximizarse la coherencia son los principios del derecho ${ }^{45}$ y las decisiones de los jueces. La decisión coherente será aquella que satisfaga en mayor medida las restricciones del derecho.

${ }^{44}$ En el sentido de falta de contenido. Este es uno de los sentidos de "forma": es lo que prescinde del contenido, "cuestión de forma pero no cuestión de sustancia". Tarello, G., op. cit., pp. 573.

45 Sin embargo, como vimos, cuáles sean estos principios y cómo deban resolverse los conflictos entre ellos de un modo que satisfaga la coherencia son cuestiones que debe aún ser clarificadas. 
Dejando de lado la ambigüedad mencionada en apartado anterior entre coherencia como proceso y producto de una forma de razonamiento y como propiedad que un sujeto epistémicamente responsable puede percibir, y la evasiva justificación de las premisas de hecho y derecho, la teoría de la coherencia bajo análisis se presenta como un modelo de justificación de decisiones jurídicas. En este sentido, parece tratarse de una teoría sobre las decisiones correctas o justificadas, pero sin contenido sustantivo, que parte de un concepto de coherencia formal.

Para Amaya el razonamiento coherentista es incompatible con una concepción instrumental de la razón práctica. Ella sostiene que el razonamiento coherentisa no es de medio a fin, sino que es un tipo de razonamiento sobre los fines o valores últimos. Sin embargo, bajo este concepto de coherencia (como propiedad) no parece justificarse por qué la coherencia (como razonamiento) consista en un tipo de razonamiento sobre fines y no sobre medios, más siendo un modelo que está desprovisto de contenido sustancial, donde expresamente se evade el problema del input (de cómo identificar las premisas) donde la justificación de la decisión parece estar dada solo en virtud de la forma del razonamiento coherentista.

Considero que, tal como la crítica de Schiavello respecto a este punto, esta concepción aparentemente formal de la coherencia no es incompatible con una racionalidad instrumenta ${ }^{46}$. Dado un sistema jurídico que establezca un principio jurídico supremo (y suponiendo que somos epistémicamente responsables), podríamos utilizar esta noción de coherencia para resolver todos los casos que se presentaran (porque justifican la finalidad del sistema y eso hace que incida en los medios para alcanzar esa finalidad).

Sin embargo, si lo que interesa es razonar sobre los fines y valores últimos, en contextos pluralistas y de conflicto reconocido la coherencia no basta. Además, hay que elegir, decidir. Sea en forma genérica, sea en el caso individual, pero en todo caso parece que hay que dar contenido (no

\footnotetext{
46 Para Schiavello esto es erróneo. Sostiene que puede reconocerse la importancia de la coherencia en el ámbito jurídico y a la vez, que haya genuinos desacuerdos prácticos. El presupuesto de la teoría de Susan Hurley que sigue Amaya es que puede en un caso individualizarse la teoría de la justicia o la teoría ética más coherente y que hay una respuesta correcta a la pregunta: ¿cómo debemos actuar en esta situación?
} 
solo determinando las restricciones propias dentro de cada domino, sino también determinando el input en base al cual se justificará una decisión).

En relación a la coherencia y la inferencia a la mejor explicación, explica Schiavello que "la coerenza può contribuire a distinguere decisioni accettabili da decisioni inaccettabili; però non consente di dimostrare che una decisione sia la migliore" 47 y que, "la domanda da porsi è se postulare l'esistenza di una risposta corretta senza esplicitare i criteri conclusivi per la sua individuazinoe abbia una qualche rilevanza pratica (di tipo esplicativo e non retorico) all'interno di una teoria del regionamento giuridico o di una teoria della prova"48, a lo que él responde en forma negativa.

\section{Naturalismo y filosofía}

Un punto que creo interesante y no ha sido considerado en el debate, pero que cada vez adquiere más importancia es el recurso al naturalismo como metodología. Es creciente en la teoría del derecho la importancia que diversos autores otorgan en este sentido, no solo a las metodologías naturalistas, sino también a la neurociencia y otras ciencias cognitivas.

Particularmente, la teoría de la coherencia aquí visitada se apoya en una metodología naturalista. Sostiene Amaya:

This model advocates a naturalistic approach to legal justification, that is, it relies on a view about the relationship between normative and descriptive issues according to which facts about how legal decision-makers reason are relevant for answering questions about how they should reason ${ }^{49}$.

47 Véase Schiavello, A., op. cit., p. 170.

48 Ibídem, p. 172. Nuevamente, es necesario aclarar que la teoría de la coherencia en esta sede analizada no se compromete con la idea de una respuesta correcta, aunque en cierta forma, no explícita, parece sugerir que hay una respuesta que goza de mayor grado de justificación que las otras (aquella que el decisor epistémicamente responsable hubiera alcanzado).

49 Véase Amaya, A., The Tapestry of Reason, op. cit., p. 545. 
La autora nos presenta un argumento basado en una forma de naturalismo a favor del razonamiento coherentista, sostiene que como así razonamos, entonces que es bueno que así justifiquemos nuestras decisiones. Afirma que el uso de criterios de coherencia es un caso natural de nuestro aparato cognitivo y que para sostener esto hay evidencia empírica. Esto le permite defender que "una razón principal a favor del coherentismo es que este goza de un alto grado de plausibilidad desde el punto de vista psicológico" ${ }^{\text {" }}$.

Amaya explica que es común asociar las cuestiones explicativas con las investigaciones científicas y las cuestiones de justificación con la filosofía. Pero que, sin embargo, la distinción en ciencia y filosofía es reciente en la historia del pensamiento y sería oportuno volver a acercarlas, como de hecho sucede en la filosofía, epistemología, ética y la filosofía de la ciencia, donde la metodología naturalista cobra cada vez más relevancia ${ }^{51}$. Defiende que:

...[A] trend towards naturalizing philosophy has been a main development in different branches of philosophy such as epistemology, philosophy of science and ethics. The main tenet of naturalism, to recall, is that philosophy is continuous with science so that empirical work has an important bearing on how the normative problems which are the main concern of philosophy should be addressed. In law, however, the naturalist trend has not taken off. With few exceptions, it continues to be assumed, among legal scholars, that issues about the psychology of legal decision-making are not relevant for answering questions about the justification of legal decisions. Thus, a strict is/ought distinction still characterizes most work in legal reasoning ${ }^{52}$.

En este paradigma naturalista se rechaza una distinción tajante entre lo normativo y lo descriptivo ${ }^{53}$, y se propugna una continuidad entre la

\footnotetext{
${ }^{50}$ Véase Amaya, A., "Diez tesis sobre la coherencia en el derecho", op. cit., p. 58.

${ }^{51}$ Véase Amaya, A., The Tapestry of Reason, op. cit., p. 550.

52 Ibídem, p. 550.

53 Ibídem, p. 550.
} 
Sobre la solución coherente de casos jurídicos

ciencia y la filosofía. De esta forma, expresamente se rechaza el argumento de la falacia naturalista:

la objeción no resulta pertinente si uno acepta una concepción naturalista del quehacer filosófico, según la cual hay una continuidad entre la filosofía y la ciencia y, en concreto, en el contexto que nos interesa, entre la filosofía y la psicología. La separación entre la filosofía y la psicología, el ámbito normativo y el descriptivo, es relativamente reciente ${ }^{54}$.

Sin embargo considero que corresponde realizar algunas consideraciones al respecto. Por un lado, no resulta del todo claro, entonces, cómo se entiende la relación entre lo normativo y lo descriptivo, el ser y el deber $\operatorname{ser}^{55}$. Si bien expresamente, en el paso antes citado, la autora niega que haya una distinción tajante entre ambos planos, es llamativo que en otras secciones de su propia construcción teórica vuelve a introducir y a hacer uso de la distinción.

Me refiero, por ejemplo, cuando Amaya enfatiza en que las razones explicativas y justificativas deben distinguirse y no pueden ser colapsadas. Ella reconoce que sobre una decisión pueden distinguirse entre razones normativas y explicativas, siendo las primeras las que determinan la corrección de la decisión y no las segundas. Al respecto afirma que una decisión sobre el derecho correcta, no dejará de serlo aunque haya sido tomada por las razones incorrectas ${ }^{56}$.

Entiendo que esto hace ver que sea necesaria una explicación de este punto y argumentar en consecuencia, ya que parece que en su propio dis-

\footnotetext{
54 Véase Ibídem, p. 59.

55 Dentro de la filosofía analítica tradicionalmente y en la teoría del derecho de corte analítica, se ha llamado la "Grand División" a la distinción entre el uso del lenguaje en función normativa o prescriptiva y el uso del lenguaje en función descriptiva o cognoscitiva, puede verse así que una de las formas de presentar la distinción se basa en la distinta función del lenguaje. Véase Guastini, R, "Cognitivismo Ludico e Regole Costitutive", en La teoria generale del diritto. Problemi e tendenze attuali, Diritto e cultura moderna 26, Comunità, Milan, 1983; Hare R.M., The Language of Morals, Clarendon Press, Oxford, 952; Ross, A, Directives and Norms, Routledge \& Kegan Paul, Londres, 1968.

56 Amaya, A., The Tapestry of Reason, op. cit., p. 525.
} 
curso la autora hace uso de una distinción categorial entre el plano explicativo y el justificativo. En caso contrario, puede reprocharse al naturalismo el confundir dos cuestiones que merecen ser consideradas en forma diferenciada respecto de la toma de decisiones en el derecho. La primera es que las decisiones son actos que están condicionados por numerosos factores y pueden ser objeto de explicación causal, psicológica, sociológica, antropológica (eventos mentales o psicológicos). Y, la segunda, es el análisis lógico-semiótico del discurso que busca describir la forma en que se presentan las razones a favor de una decisión (premisas de un razonamiento, razones que justifican una tesis) $)^{57}$.

Por otro lado, respecto de la alegada la continuidad entre filosofía y ciencia, podemos precisar que la distancia o continuidad entre la ciencia y la filosofía no depende del objeto de estudio como parece sugerirse. En este sentido, no es que la filosofía dejara de ocuparse de cuestiones como la acción o la mente, sino que las preguntas que se hacen desde cada perspectiva son categorialmente diferentes.

Es en este sentido que, por ejemplo, Hacker critica al Naturalismo ${ }^{58}$. El Naturalismo, con base en Quine y su crítica a la distinción analitico/ sintético, sostiene que no hay lugar para el análisis conceptual y que, por lo tanto, la actividad filosófica sería una continuación de la actividad científica. Hacker, específicamente en relación a la epistemología naturalista, señala que esta no puede distinguir entre distintos tipos de conocimientos (por ejemplo, entre el conocimiento social y natural, entre el conocimiento en matemáticas y el conocimiento de un amigo, etc.).

Lo que el filósofo busca comprender es cuándo el conocimiento requiere o no justificación; qué entendemos cuando decimos que alguien tiene conocimiento; si se trata de un estado mental, de un logro, un acto, una disposición, una habilidad; si el conocimiento es igual a un estado de nuestro cerebro; por qué podemos decir que 'alguien cree que $p$, pero no es el caso que $p$ ', pero no podemos decir que 'yo creo que $p$ pero no es

${ }^{57}$ Respecto a la distinción entre razonamiento como proceso mental y como discurso véase Guastini, R., Interpretar y Argumentar, op. cit., p. 225.

${ }^{58}$ Hacker específicamente critica los naturalismos epistemológicos, ontológico y filosófico. Véase Hacker, P.M.S., "Passing by the Naturalistic Turn: On Quine’s Cul-de-Sac", Philosophy, 81 (316), 2006. 
Sobre la solución coherente de casos jurídicos

el caso que $p^{\prime}$; por qué hay métodos para adquirir conocimiento pero no creencias; etc. Todas estas cuestiones no pueden responderse desde una epistemología naturalizada.

Explica que:

\begin{abstract}
"What needs to be clarified if these questions are to be answered is the web of our epistemic concepts, the ways in which the various concepts hang together, the various forms of their compatibilities and incompatibilities, their point and purpose, their presuppositions and different forms of context dependency. To this venerable exercise in connective analysis, scientific knowledge, psychology, neuroscience and self-styled cognitive science can contribute nothing whatsoever. ${ }^{59}$
\end{abstract}

Este argumento, aplicado al tema específico que nos compete, requeriría que una teoría general de la coherencia explique el marco o esquema conceptual en el que la coherencia se ubica. Es decir cómo se relaciona la coherencia con la interpretación, cómo funcionan los argumentos que tienen a la coherencia como base, la coherencia entre normas de igual jerarquía, de diversa jerarquía, de sistemas de normas diversos, cómo se relaciona la coherencia con la verdad, con la justificación, con el concepto de racionalidad ${ }^{60}$, con los conceptos de consistencia y la idea de que los sistemas jurídicos son completos ${ }^{61}$, con el concepto de consistencia y unidad del sistema ${ }^{62}$, con el concepto de agencia, de comunidad, y, sobre todo, ¿de qué se predica la coherencia? Estas cuestiones, relevantes para clarificar los sentidos en que usamos "coherencia", parecen ser los temas ineludibles para futuras investigaciones en la materia ${ }^{63}$.

59 Ibídem, p. 247.

${ }^{60}$ Sobre el que han indagado Atienza, Michelon y Lariguet en sus respectivos comentarios.

${ }^{61}$ Al respecto han trabajado Ratti, , G.B. y Rodríguez, J.L., "On Coherence as a Formal Property of Normative Systems", Revus, 27, 2015, pp. 131-146.

62 C. Michelon C., "Las Razones de la Coherencia", Discusiones, X, 2010.

${ }^{63}$ Este tipo de investigaciones conceptuales contribuyen principalmente a comprender lo que se sabe y dar mayor claridad respecto de las preguntas sobre lo que no se conoce. Al 
Es importante resaltar que esto no implica negar la importancia y utilidad de los estudios empíricos. Sino puntualizar que las preguntas o cuestiones filosóficas en relación a nuestros esquemas conceptuales son categorialmente diversas. El filósofo trabaja con conceptos que busca clarificar, intenta explicitar las presuposiciones del uso de los conceptos e indagar sobre el propósito de tener esa expresión en el lenguaje. En este sentido, Hacker explica que la filosofía no busca decirle a los científicos que estudiar y qué no, o cómo diseñar experimentos. Lo que el filósofo puede hacer es un examen de la forma en que usamos los conceptos al establecer un determinado problema, al diseñar un experimento o al interpretar los resultados de estos experimentos o de las observaciones.

La importancia y utilidad de los estudios empíricos debe ser contextualizada y, por sobre todo, el enfoque o metodología "naturalista" debe aun fundamentarse. La fundamentación es más que relevante si, como sostiene Amaya, estos estudios empíricos sirven para ver cómo resolver problemas normativos. Especialmente, en este caso particular, dada la relevancia práctica que para el estado de derecho tiene la justificación de decisiones jurídicas.

\section{Una super-teoría de la coherencia}

Se ha señalado cómo el término coherencia es usado para hacer referencia a varias $\operatorname{cosas}^{64}$, y que esto es un obstáculo que no ayudada a la comprensión de la teoría, y obstruye la capacidad de explicar y justificar la toma de decisiones en el derecho.

Me parece oportuno llamar la atención sobre un ejemplo que MacCormick plantea en el apéndice de su Legal Reasoning and Legal Theroy ${ }^{65}$, respecto a cómo comprender el punto de vista interno de las reglas planteado por Hart en El concepto de Derecho. MacCormick nos recuerda Los Viajes

respecto véase, Bennett, M. y Hacker, P., Neuroscience and Philosophy: Brain, Mind, and Language, New York, Columbia University Press, 2007, p. 46.

64 Véase Atienza, M., “¿Coherencia o racionalidad?”, Discusiones, X, 2010.

65 Véase MacCormick, N., "Appendix: On the 'Internal Aspect' of Norms" en Legal Reasoning and Legal Theory, Oxford, Oxford University Press, 1978, p. 275. 
Sobre la solución coherente de casos jurídicos

de Gulliver cuando Gulliver es encontrado en las costas de Lilliput y el rey ordena que sea registrado:

De la faltriquera de la derecha colgaba una gran cadena de plata, con una extraordinaria suerte de máquina al extremo. Le instamos a que sacara lo que hubiese sujeto a esa cadena, que resultó ser una esfera la mitad de plata y la otra mitad de un metal transparente, porque en el lado transparente vimos ciertas extrañas cifras, dibujadas en círculo, y que creímos poder tocar, hasta que nos dimos cuenta de que nos detenía los dedos aquella sustancia translúcida. Nos acercó a los oídos este artilugio, que producía un ruido incesante, como el de un molino de agua. Supusimos que es, o algún animal desconocido, o el dios al que venera; aunque nos inclinamos por lo último, porque nos aseguró (si es que lo entendimos bien, pues se expresaba muy imperfectamente) que rara vez hacía nada sin consultarlo. Lo llamaba su oráculo, y dijo que señalaba cuándo era el momento de cada una de las acciones de su vida ${ }^{66}$.

La mejor explicación que los liliputienses pudieron hacer respecto a qué era aquello que Gulliver guardaba en su bolsillo consistía en que ese objeto era el dios al que él adoraba. Esta era una explicación coherente, daba un sentido a los lilliputienses para atribuir a ese objeto. Sin embargo, aun esta explicación coherente requiere explicitar el punto de vista, es decir el "input" aceptado. Nosotros rechazaríamos esta explicación de los liliputienenses porque, aunque sea coherente, usamos y somos parte de la práctica de usar relojes para calcular el paso del tiempo. En este sentido, si bien la explicación en cuestión podría ser de utilidad para los liliputienses, no es la mejor explicación desde nuestro punto de vista.

En el ejemplo propuesto por MacCormick, en donde los liliputienses encuentran a Gulliver en posesión de un objeto que desconocen e interpretan que este es "el dios que él adora", los liliputienses no contaban con el punto de vista interno respecto de las reglas que hacen posible comprender 
qué es y en qué consiste usar un reloj ${ }^{67}$. En el mismo sentido, las explicaciones antropológicas pueden ser variadas y coherentes en relación a los sentidos previamente aceptados por el investigador. Hay un importante punto en que los sentidos deben ser aquellos importantes para los participantes, para lo cual es necesario aprender las prácticas en cuestión, su point, su finalidad, y la idea de coherencia no podría estar exenta de esto ${ }^{68}$. Amaya reconoce en parte este punto. Por un lado, dando importancia al contexto y, por otro lado, al reconocer que pueden presentarse diversos modelos coherentes para explicar la misma cosa. Es nuevamente la responsabilidad epistémica la que permitiría optar entre diversas explicaciones coherentes ${ }^{69}$. Pienso que, otra forma de resolver esta elección, que no implica esconderla, es simplemente hacerla explícita. Lo que debe

${ }^{67}$ MacCormick, N., op. cit., p. 288.

${ }^{68}$ Los problemas al respecto se han presentado específicamente en el ámbito de la antropología social. Al respecto explica por ejemplo Jori, como esto se relaciona con la idea de que corresponde descubrir la racionalidad interna de determinados comportamientos que, a simple vista, para el observador externo parecen irracionales, para la descripción y explicación antropológica de comportamientos en una sociedad distinta a la propia (en su texto el habla de una sociedad "primitiva"). Esta idea encuentra su base en el positivismo lógico o neopositivismo que entiende que los sistemas lingüísticos y conceptuales tienen fundamento convencional y, por eso, admiten que tengan un grado de racionalidad interna. Véase Jori, M., "Il giurista selvaggio en Un contributo alla metodologia della descrizione sociale", Sociología del Diritto, 1, 1974, Milán, Giuffrè, 1974, pp. 85-108. Debe notarse que, más allá del positivismo lógico y la idea de convenciones, la misma idea puede entenderse, siguiendo la obra de Wittgenstein en sus investigaciones filosóficas (en relación a la comprensión del lenguaje) y la interpretación que de él hace Peter Winch (y aplica a la comprensión de prácticas sociales), como sentidos que dependen de una específica forma de vida, de una práctica o juego del lenguaje particular. Véase Wittgenstein, L., Investigaciones Filosóficas, Altaya, España, 1999 [1953] y Winch, P., The Idea of a Social Science and its Relation to Philosophy, Routledge, Londres, 2008 [1958].

${ }^{69}$ Sobre este punto, respecto a los límites de la coherencia, Michelon distingue entre dos problemas, relacionados, pero diversos. El primero, sobre cómo evitar el abuso subjetivo de las estrategias de coherencia y, el segundo, relacionado a los criterios de racionalidad que nos permiten juzgar cuál de dos posibles explicaciones es racionalmente superior. Y, con razón, considera que a estos fines no es suficiente la noción de sujeto virtuoso. Véase Michelon, C., "Las razones de la coherencia", Discusiones, X, 2010. 
realizarse es la explicitación del punto de vista desde el que se coherentiza, del contexto y, como también aceptaría Amaya, del sujeto coherentizador ${ }^{70}$.

Como vimos, la toma de decisiones en el derecho requiere justificar estas decisiones conforme a derecho, determinando que el punto de vista relevante para determinar la coherencia es aquel del ordenamiento jurídico en cuestión, por lo tanto coherentizar el derecho desde un punto de vista no jurídico podría llevarnos a un problema. Respecto de la coherencia (y no solo) es importante explicitar los puntos de vista, los presupuestos, los valores o principios desde donde se identifican normas, se interpretan los hechos y se toman decisiones jurídicas. Lo relevante es explicitar el dominio, es decir con cuáles elementos estamos haciendo coherente nuestra decisión. Podemos concluir que aun aceptando la relevancia de la coherencia, esta no justifica de por sí una decisión ${ }^{71}$. Desde ya, sería tal vez una tarea insensata buscar la justificación última del input de cada dominio, por esto podríamos acordar que lo importante es al menos explicitarlo.

En estas páginas he tratado más que nada de problematizar uno de los aportes más exhaustivos respecto de la coherencia en el derecho. Ha sido un intento de retomar una rica discusión y proponer por dónde esta podría avivarse, como quien dice: he intentado echar más leña al fuego.

\section{Bibliografía}

Alexy R., Teoría de los derechos fundamentales, Madrid, Centro de Estudios Constitucionales, 1993 [1986]

${ }^{70}$ La sugerencia de explicitar al punto de vista del agente podría entenderse, dentro de la propuesta de Amaya, como otro criterio de responsabilidad epistémica, junto con los que ella menciona: la diligencia, la valentía para enfrentar la crítica, la perseverancia, o la apertura de mente. Véase Amaya, A., "Diez tesis sobre la coherencia en el derecho", op. cit., p. 6.

${ }^{71}$ Entiendo que es en esta dirección que Schiavello discutía: "la maggiore coerenza di una storia rispetto ad unaltra viene necessariamente valutata (anche) a partire da una serie di preferenze personali di vario genere che sfuggono ad una valutazione di tipo razionale. Ancora una volta, questo significa che non esiste la spiegazione migliore, ma molteplici spiegazioni accettabili, e che il prevalere dell'una rispetto alle altre dipende anche dai presupposti di partenza" Schiavello, A., "Tre osservazioni su Diez Tesis acerca de la coherencia en el derecho", op. cit., p. 172. 
Alonso, J.P., Principios Jurídicos Implícitos, Madrid, Marcial Pons, 2018.

Alonso, J.P., "Principios jurídicos implícitos y coherencia”, Doxa, 36, 2013, pp. 375-385.

Amaya, A., "Diez tesis sobre la coherencia en el derecho", Discusiones, X, 2010, pp. 21-64.

Amaya, A., "Coherencia, justificación y derecho. Respuestas a los participantes", Discusiones, X, 2010, pp. 216-264.

Amaya, A., The Tapestry of Reason. An inquiry into the Nature of Coherence and its Role in Legal Argument, Oxford, Hart Publishing, 2015.

Atienza, M., “¿Coherencia o racionalidad?”, Discusiones, X, 2010, pp. 11-20.

Bennett, M. y Hacker, P., Neuroscience and Philosophy: Brain, Mind, and Language, Nueva York, Columbia University Press, 2007.

Guastini, R, "Cognitivismo Ludico e Regole Costitutive" en La teoria generale del diritto. Problemi e tendenze attuali, Diritto e cultura moderna 26, Milan, Comunità, 1983.

Guastini, R., Interpretar y Argumentar, Madrid, Centro de Estudios Políticos y Constitucionales, 2014.

Hacker, P.M.S., "Passing by the Naturalistic Turn: On Quine's Cul-de-Sac", Philosophy, 81 (316), 2006, pp. 231-253.

Hare, R.M., The Language of Morals, Oxford, Clarendon Press, 952.

Hart, H.L.A., El concepto de derecho, Buenos Aires, Abeledo-Perrot, 2011. Hart, H.L.A., "Postscriptum" en Bonilla, D., et al. La Decisión Judicial. El debate Hart-Dworkin, Bogotá, Universidad de los Andes, Siglo del Hombre Editores, 1997, pp. 89-141.

Jori, M., "Il giurista selvaggio en Un contributo alla metodologia della descrizione sociale”, Sociología del Diritto, 1, 1974, Milán, Giuffrè, 1974, pp. 85-108.

Kelsen, H., Teoría Pura del derecho, Buenos Aires, Ediciones Colihue, 2011.

MacCormick, N., “Appendix: On the 'Internal Aspect' of Norms" en Legal Reasoning and Legal Theory, Oxford, Oxford University Press, 1978.

Marmor, A., Interpretation and Legal Theory, 2da ed., Hart Publishing, 2005.

Michelon, C., "Las razones de la coherencia”, Discusiones, X, 2010, pp. 139153. 
Navarro, P. y Rodríguez J.L., Deontic Logic and Legal Systems, Cambridge, Cambridge Univertisy Press, 2014.

Pérez Bermejo J.M., "Alcune osservazioni sul valore della coerenza nei sistemi giuridici”, Diritto \& questioni pubbliche, 7, 2007, pp. 43-59.

Ratti, G.B., "La coerentizzazione dei sitemi giuridici", Diritto \& questioni pubbliche, 7, 2007, pp. 61-70.

Ratti, G.B. y Rodríguez J.L., “On Coherence as a Formal Property of Normative Systems", Revus, 27, 2015, pp. 131-146.

Redondo, M.C., "Decidir en modo justificado: una explicación coherentista”, Discusiones, X, 2010, pp. 65-86.

Ross, A, Directives and Norms, Londres, Routledge \& Kegan Paul, 1968.

Schiavello, A., "Tre osservazioni su Diez Tesis acerca de la coherencia en el derecho", Discusiones, X, 2010, pp. 155-178.

Tarello, G., "Formalismo", Novissimo digesto italiano, Torino, UTET, vol. VII, pp. 571-580.

Thagard, P. y Verbeurgt, K., "Coherence as Constraint Satisfaction", Cognitive Science, 22 (1), 1998, pp. 1-24.

Wróblewski, J., "Justificación de las decisiones jurídicas” en Sentido y hecho en el derecho, México, Fontamara, 2001 [1979], pp. 45- 65.

Wróblewski, J., "La interpretación en el derecho: teoría e ideología" en Sentido y hecho en el derecho, México, Fontamara, 2001 [1979], pp. 149- 171.

Wróblewski, J., The judicial aplication of the law, ed. por Bankowski, Z. y MacCormick, N., Kluwer, Dordrecht, 1992 [1972].

Winch, P., The Idea of a Social Science and its Relation to Philosophy, Londres, Routledge, 2008 [1958].

Wittgenstein, L., Investigaciones Filosóficas, Barcelona, Altaya, 1999 [1953]. 